\title{
Do Deferoxamine, Ticlopidin or Trimetazidine Prevent Sevoflurane Nephrotoxicity? An Electron Microscopic Study
}

\author{
Karamanlioglu B* and Aktas $\mathrm{RG}^{* *}$ \\ *Trakya University, Faculty of Medicine, Edirne, TURKEY \\ **Zonguldak Karaelmas University, Faculty of Medicine, Zonguldak, TURKEY
}

\section{Introduction}

Sevoflurane is a common anesthetic drug. Several biochemical, pharmacologic and physiologic studies have showed nephrotoxicity of Sevoflurane[1,2,3]. Histopathologic studies reported tubular damage and tubular cellular hyperplasia after the administration of Sevoflurane[4]. Deferoxamine(DFO) is known to protect against myoglobinuric acute renal failure[5]. It has been suggested that Ticlopidine(TIC) does not prevent nephropathies but has beneficial effects[6]. Fang et al. showed that TIC inhibited mesangial cell proliferation and collagen synthesis[7]. There is another study reporting that TIC induces acute interstitial nephrite and cause an increase of the number of lymphocytes and eosinophil leucocytes in interstitial tissue[8].

Trimetazidine(TMZ) has anti-ischemic effects and protects tissue damage in kidney[5, 9, 10, 11]. These studies lead us to investigate if DFO, TIC or TMZ can prevent the nephrotoxicity of Sevoflurane at morphologic level.

\section{Materials\&Methods}

Fifty male Wistar-Albino rats were used for this experimental study. They were divided into five groups randomly: Group I was control and had $1.5 \mathrm{cc}$ saline two times a day. Group II had $15 \%$ Oxygen $+85 \%$ Nitricoxide $+3 \%$ Sevoflurane in a special designed chamber for 1 hour. Group III had i.m. $15 \mathrm{mg}$ DFO(Desferal, Novartis) for 7 days and then they inhaled the same Sevoflurane combination as Group II for 1 hour. Group IV had oral $25 \mathrm{mg}$ TIC(Ticlid, Sanofi) that was dissolved in $1.5 \mathrm{cc}$ saline. After that, they had same Sevoflurane combination for 1 hour. Group V had oral 0,5 mg TMZ(Vastarel, Servier) that was dissolved in 1,5 cc saline. They also inhaled Sevoflurane combination at the same way with the other groups.

Biopsy specimens from renal cortex of each rat were embedded in Araldite and the ultrathin sections were examined under transmission electron microscope.

\section{Results}

Electron microscopic findings were as the follows:

Group I : Glomeruli, proximal tubules and distal tubules showed normal electron microscopic features.

Group II: Basement membranes of proximal and distal tubules were irregular. The relationships between the cells and the basement membranes were decreased. The foldings at the basal side of the cells were diminished. Frequency of the microvilli on the apical surface of the proximal cells was less. There were gaps between the epithelial cells. Basement membranes of glomeruli were regular. Pedicels of podocytes were irregular at some places. Mesangial matrix increased slightly. Nuclear chromatin of some endothelial cells, podocytes and mesangial cells were condensed. Group III: The electron microscopic findings in tubular cells were similar to the findings, which were observed in Group II. However, they were less significant. There were no changes in glomeruli. 
Group IV: The main significant ultrastuctural finding in tubular epithelial cells was the increase of foldings at the basal side. Fine structure of glomeruli showed no abnormalities.

Group V: The foldings at the basal side of proximal and distal tubular cells were increased. There were big gaps between the epithelial cells. The raise in the number of lysosomes were evident. There were some changes in glomeruli, too: Mesangial matrix was increased. Pedicels of podocytes lost their regularity partly.

\section{Conclusions}

Tubular cells are very sensitive to ischemic damage. Our observations after the administration of Sevoflurane reflect the reversible tubular findings, which were seen after ischemia. Irreversible changes after ischemia were not clear at this experiment. Either DFO or TIC caused diminishment of the microscopic findings. We conclude that the anti-oxidant effects of these drugs might be useful to prevent nephrotoxicity of Sevoflurane. Fine structural changes after the treatment with TMZ were similar to the findings observed after Sevoflurane administration. This suggests that TMZ was not successful to prevent the nephrotoxicity of Sevoflurane. Our findings related with DFO and TIC are consistent with the previous studies. However, the results of this study did not show beneficial effects of TMZ as it has been reported previously at clinical studies. Attemption of different dosages and different duration times for the application of this drug with Sevoflurane might be helpful to verify if TMZ can prevent Sevoflurane nephrotoxicity.

\section{References}

[1]Conzen PF et al. Anesthesiology 97(2002)578.

[2]Ebert TJ and Arain SR. Anesthesiology 93(2000)1401.

[3]Obata R et al. Anesth Analg 91(2000)1262.

[4]Keller KA et al. Anesthesiology 83(1995)1220.

[5]Chander V et al. Pharmacology 67(2003) 41.

[6]Riyuzo MC and Soares VA. Ren Fail 21(1999) 469.

[7]Fang CC et al. J Formos Med Assoc 97(1998) 458.

[8]Rosen H et al. Am J Kidney Dis 25(1995)934.

[9]Sucu N et al. Pharmacol Res 46(2002)345.

[10]Satyanarayana PS and Chopra K. Ren Fail 24(2002)259.

[11]Baumert et al. Transplantation 68(1999) 300.

[12]Ozden A et al. Eur Surg Res 30(1998) 227. 